\title{
Laboratory spectroscopy and astronomical significance of the fully-benzenoid PAH triphenylene and its cation
}

\author{
V. Kofman ${ }^{a, b, *}$, P.J. Sarre ${ }^{c}$, R.E. Hibbins ${ }^{c, d}$, I.L. ten Kate ${ }^{b}$, H. Linnartz ${ }^{a}$ \\ a Sackler Laboratory for Astrophysics, Leiden Observatory, Leiden University, PO Box 9513, 2300 RA Leiden, The Netherlands \\ ${ }^{\mathrm{b}}$ Department of Earth Sciences, Utrecht University, Budapestlaan 4, 3584 CD Utrecht, The Netherlands \\ ' School of Chemistry, The University of Nottingham, University Park, Nottingham NG7 2RD, United Kingdom \\ ${ }^{\mathrm{d}}$ Department of Physics, Norwegian University of Science and Technology, N-7491 Trondheim, Norway
}

\section{A R T I C L E I N F O}

\section{Article history:}

Received 18 January 2017

Revised 19 April 2017

Accepted 19 April 2017

Available online 20 April 2017

\section{Keywords:}

Astrochemistry

Molecular processes

Methods: laboratory

Techniques: spectroscopic

ISM: molecules

\begin{abstract}
A B S T R A C T
Triphenylene $\left(\mathrm{C}_{18} \mathrm{H}_{12}\right)$ is a highly symmetric polycyclic aromatic hydrocarbon (PAH) molecule with a 'fully-benzenoid' electronic structure. This confers a high chemical stability compared with PAHs of similar size. Although numerous infrared and UV-vis experimental spectroscopic and theoretical studies of a wide range PAHs in an astrophysical context have been conducted, triphenylene and its radical cation have received almost no attention. There exists a huge body of spectroscopic evidence for neutral and ionised PAHs in astrophysical sources, obtained principally through detection of infrared emission features that are characteristic of PAHs as a chemical class. However, it has so far not proved possible to identify spectroscopically a single isolated PAH in space, although PAHs including triphenylene have been detected mass spectrometrically in meteorites. In this work we focus on recording laboratory electronic spectra of neutral and ionised triphenylene between 220 and $780 \mathrm{~nm}$, trapped in $\mathrm{H}_{2} \mathrm{O}$ ice and solid argon at $12 \mathrm{~K}$. The studies are motivated by the potential for spectroscopic astronomical detection of electronic absorption spectra of PAHs in ice mantles on interstellar grains as discussed by Linnartz (2014), and were performed also in a cold $\mathrm{Ar}$ matrix to provide guidance as to whether triphenylene (particularly in its singly positively ionised form) could be a viable candidate for any of the unidentified diffuse interstellar absorption bands. Based on the argon-matrix experimental results, comparison is made with previously unpublished astronomical spectra near $400 \mathrm{~nm}$ which contain broad interstellar absorption features consistent with the predictions from the laboratory matrix spectra, thus providing motivation for the recording of gas-phase electronic spectra of the internally cold triphenylene cation.
\end{abstract}

(C) 2017 Elsevier B.V. All rights reserved.

\section{Introduction}

Polycyclic aromatic hydrocarbons (PAHs) are ubiquitous in space and observed in many types of astrophysical environments (Tielens, 2013). Their presence is inferred from a number of midinfrared emission features at wavelengths that are characteristic of C-C and C-H vibrational modes of PAHs (Allamandola et al., 1989).

It is generally accepted that excited vibrational levels of PAHs emit infrared radiation on relaxation after electronic excitation, for example in photon dominated regions (Tielens, 2013). The physical and chemical processes at play on excitation are currently a topic of intense laboratory study; PAHs can be ionized or dissociate on photon absorption (Zhen et al., 2014a; 2015), which holds the potential to enrich the interstellar medium with charged hydro-

\footnotetext{
* Corresponding author.

E-mail addresses: kofman@strw.leidenuniv.nl (V. Kofman), peter.sarre@nottingham.ac.uk (P.J. Sarre).
}

carbons and larger hydrocarbon species that are hard to generate through bottom-up reaction schemes. Recently, it was shown experimentally that it is possible to form $\mathrm{C}_{60}$ by (multi-photon) photolysis of very large PAHs (Zhen et al., 2014b), in line with model predictions for astronomically more relevant excitation schemes (Berné et al., 2015; 2016). The molecules $C_{60}, C_{70}$ and $C_{60}{ }^{+}$have been observed in a number of astrophysical sources through their IR emission spectra (Berné et al., 2013; Cami et al., 2010; Sellgren et al., 2010). More recently, Campbell et al. (2015) have claimed the assignment of two stronger and possibly three more weaker diffuse interstellar absorption features in the 900-1000 nm region, as due to electronic transitions of $\mathrm{C}_{60}{ }^{+}$(Campbell et al., 2015; 2016a; 2016b; Walker et al., 2015). Attempts to compare optical laboratory data for gas-phase spectra of PAHs with DIB absorption features recorded through diffuse interstellar clouds have not led to identification of any PAH as a DIB carrier. A wide range of PAHs could be present including neutral, ionised, protonated, deprotonated, hydrogenated, dehydrogenated and substituted forms, 


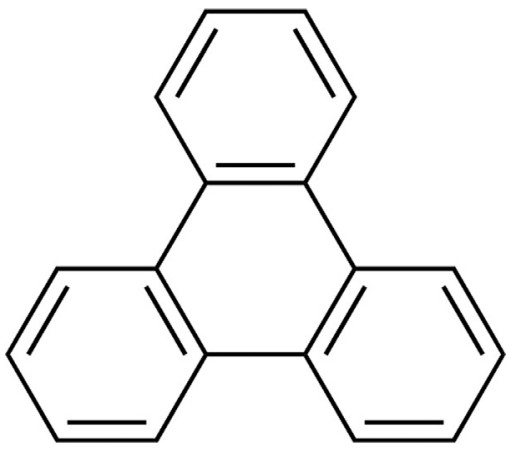

Fig. 1. Molecular structure of triphenylene $\left(\mathrm{C}_{18} \mathrm{H}_{12}\right)$. The peripheral hydrogen atoms are omitted for clarity.

however not all of these have been explored experimentally. Surveys and critical assessments of the issues have been presented by Salama (2008) and references therein (Gredel et al. (2011); Huisken et al. (2014); Salama and Ehrenfreund (2014); Salama et al. (2011); Steglich et al. (2011). Neutral triphenylene, $\mathrm{C}_{18} \mathrm{H}_{12}$, is a highly symmetric $\left(D_{3 h}\right)$ molecule containing three aromatic rings (see Fig. 1). It is a fully benzenoid PAH, which distinguishes it from many other PAHs as its aromatic rings are directly connected to each other, and all carbon atoms participate in aromatic stabilization. As benzenoid PAHs are both chemically and photochemically less reactive than non-benzenoid PAHs, this makes triphenylene particularly interesting in an astronomical context, and its cation as a potential DIB carrier. Triphenylene has been found in isomer-specific mass spectra of meteorite samples (Callahan et al., 2008) and contains six hydrogen atoms in 'bay' sites. Based on the interpretation of the line shapes of IR emission features, PAHs with such sites are thought to be present in the ISM (Candian et al., 2010).

The outline of this paper is as follows. In the next section the motivation for the experiments is discussed and the experimental details are given in Section 3. Section 4 summarizes what is known about triphenylene and its cation in the literature. This information is linked with the experimental data in Section 5. The final section concludes with a discussion of the astronomical relevance of these findings.

\section{Experimental-astronomical motivation}

\subsection{PAHs in water ice}

PAHs are expected to freeze out, as do many other volatile species, on cold dust grain in interstellar and circumstellar media. As water forms the main constituent of ice in space, the study of PAHs in water ice is of particular interest. In the laboratory, where specific PAHs can be selected, IR and UV-vis studies have provided much information on the physical and chemical properties of such species embedded in water ice (e.g. Bernstein et al. (2005, 2007); Bouwman et al. (2011); Cuylle et al. (2014); Guennoun et al. (2011); Sandford et al. (2004)). In astronomical infrared ice surveys, however, many PAHs have similar vibrational modes, causing spectral features to overlap. It is here that optical electronic solid state spectra of PAHs in a low temperature water matrix can offer an attractive potential alternative (Linnartz, 2014). Water itself does not absorb in the UV-vis, and a significant number of electronic transitions of neutral and ionised PAHs have much higher transition strengths and are more molecule-specific than their vibrational spectra. This means that in laboratory studies of optical spectra highly diluted mixtures of PAHs embedded in water ice can be used, which are more representative of real astronomical conditions. Optical absorption spectroscopy of PAHs embedded in interstellar ice analogues was introduced in 2003 by Gudipati and Allamandola (Gudipati and Allamandola, 2003; 2004; 2006) and has more recently been applied to a number of cases in Leiden (Bouwman et al., 2011; 2009; Cuylle et al., 2014). A range of PAHs embedded in various matrix environments (mainly $\mathrm{H}_{2} \mathrm{O}, \mathrm{NH}_{3}$ and $\mathrm{Ne} / \mathrm{Ar}$ ) has been studied, along with their spectroscopic dependence on a number of parameters, including temperature, ice morphology and concentration. The present study adds to this research through the study of triphenylene and its cation embedded in water ice. With the discovery that radical cations of PAHs can be stable in water ice for extended periods of time, i.e. more than several weeks (Gudipati and Allamandola, 2006), the potential astrochemical relevance of these trapped ions became clear. This same study showed that up to $70 \%$ of the embedded neutral PAH sample can be ionised by VUV radiation. As the electrons and radical cations remain separated in the ice, both species can participate in subsequent reactions. Regeneration of the parent neutral PAH is not significantly observed on the heating of ice-embedded PAH cations (Gudipati and Allamandola, 2006). Recent IR studies show the formation of alcohols $(\mathrm{PAH}-\mathrm{OH})$ and ketones $(\mathrm{PAH}=\mathrm{O}$ ) (Cook et al., 2015; Guennoun et al., 2011) in vacuum UV (VUV) irradiated PAHcontaining water ices at $14 \mathrm{~K}$ temperatures. Generally observed is that critical to the lifetime of the radical ion is the morphology of the ice where, upon heating, the transition from amorphous to crystalline ice initiates the end of its lifetime. Up to that point, the stability argument suggests that astrophysical ices may be enriched with ions, and, as the main constituents of ice are transparent to visible light, these species may thus be detected by their absorption features in direct or scattered light.

\subsection{PAHs as diffuse band carriers}

PAHs have been discussed as potential carriers of some, possibly many, of the diffuse interstellar absorption bands for many years. However, to date none of the PAH neutrals, radical cations or protonated PAHs that have been studied spectroscopically in matrices or in the gas-phase has been found to have a definitive correspondence with spectra observed towards reddened background stars. It is clear that the strongest diffuse bands do not originate in small $\mathrm{PAH}$ species, but this does not rule out PAHs as an entire class contributing to interstellar absorption. The recent claim of $\mathrm{C}_{60}{ }^{+}$as a diffuse band carrier by Campbell et al. (2015) using dissociation spectroscopy of small helium tagged $\mathrm{C}_{60}{ }^{+}$-complexes was built initially on matrix absorption experiments by Fulara et al. (1993) that are very similar to those reported here for the triphenylene cation $\left(\mathrm{C}_{18} \mathrm{H}_{12}{ }^{+}\right)$. The matrix results inspired astronomical observations by Foing and Ehrenfreund (1994) in the relatively unexplored and observationally challenging spectral region near $950 \mathrm{~nm}$ which revealed new diffuse bands in possible correspondence with the matrix data of Fulara et al. (1993). The recent recording of complementary gas-phase spectra of $\mathrm{He}_{n} \mathrm{C}_{60}+$ Kuhn et al. (2016), confirms the laboratory rest wavelengths published Campbell et al. (2015, 2016a). Currently, several astronomical groups focus on the interpretation of the astronomical data that are situated in a wavelength domain where telluric pollution by water features causes a serious issue (Cordiner et al., 2017; Galazutdinov et al., 2017; Walker et al., 2016).

\section{Experimental details}

The spectra of triphenylene and its cation were measured using OASIS, our Optical Absorption Setup for Ice Spectroscopy. The setup has been used in a number of comparable studies previously, and details are available in Bouwman et al. (2009) and Allodi et al. (2013). Dilute ice mixtures of PAH and water/argon (between $1: 3,000$ and $1: 8,000$ ) were prepared by sublimating triphenylene and co-depositing this with $\mathrm{H}_{2} \mathrm{O}$ or Ar onto a UV-vis-transparent 
$\mathrm{MgF}_{2}$ window held at $12 \mathrm{~K}$. The sample is mounted in a high vacuum chamber $\left(10^{-7}\right.$ mbar) on top of a cold finger that is cooled by a closed cycle helium cryostat. Absolute temperature control is realized through resistive heating using a Lakeshore temperature controller. The resulting ice thickness is determined by laser interferometry in the reflected light of a HeNe laser; a rather common procedure. See Baratta and Palumbo (1998) and for a recent overview Bossa et al. (2014). Typical ice thicknesses studied here are between 1 and $2 \mu \mathrm{m}$. In these experiments the number of triphenylene molecules in the sample was determined by integrating the absorbance of the $S_{3} \leftarrow S_{0}$ transition $(227-268 \mathrm{~nm}$; $\mathrm{f}=0.99$ ) and using the conversion factor $8.85 \times 10^{-13}$ from oscillator strength to $\mathrm{cm}$ molecule ${ }^{-1}$ as described by Bouwman et al. (2009); Hudgins et al. (1993); Kjaergaard et al. (2000). A commercial sample of triphenylene (98\% purity) was used without further purification. It was heated typically to a temperature of $350 \mathrm{~K}$ in a small oven positioned close to the substrate holder. For the matrix, ultrapure milli-Q generated water was degassed in several freezepump-thaw cycles prior to use; commercial argon 5.0 was used without further purification. Spectra were taken using a deep UV xenon-arc lamp that covers the wavelength range 220-1200 nm. The light was guided through the ice sample and after dispersion by a spectrograph, collected on a CCD camera. The spectral resolution is $0.6 \mathrm{~nm}$ per pixel, but higher resolution spectroscopy can be realized by using different gratings. Given the broad nature of the solid state spectra recorded here, a higher resolution was not needed. To generate ions, a $\mathrm{H}_{2}$ microwave discharge lamp was used which generates mainly Ly- $\alpha$ and $160 \mathrm{~nm}$ VUV photons, with a typical flux of $1-5 \times 10^{13}$ photons $\mathrm{cm}^{-2} \mathrm{~s}^{-1}$ at the ice sample (Ligterink et al., 2015; Warneck, 1962). Electronic spectra of the cations were taken by monitoring the absorption with respect to the non-irradiated ice. Positive features are thus the formed reaction products (i.e., cation) and a negative feature illustrates the consumption of a precursor species. The spectra shown are the result of the addition of 100-200 spectra, or 2-4 seconds of integration depending on the settings. Whereas in previous studies (e.g. Bouwman et al., 2011) the $\mathrm{PAH}$ and $\mathrm{PAH}^{+}$spectral features are generally well separated, this is not the case for the triphenylene system below $300 \mathrm{~nm}$. As a result the cation spectra below $290 \mathrm{~nm}$ are not shown or discussed.

\section{Spectroscopy of triphenylene}

Gas-phase $\left(S_{1}-S_{0}\right)$ spectra of neutral triphenylene recorded by laser-induced-fluorescence and dispersed fluorescence spectroscopy have been reported (Kokkin et al., 2007). The origin band transition is symmetry-forbidden and the strongest vibronically allowed band $\left(35_{0}^{1}\right)$ near $329 \mathrm{~nm}$ is calculated to have an extremely low f-value of 0.0007 (Kokkin et al., 2007). In a recent sensitive study of the 305-370 nm region no interstellar absorption was found at this wavelength (Bhatt and Cami, 2015). The $S_{2} \leftarrow S_{0}$ and $S_{3} \leftarrow S_{0}$ transitions are much stronger with oscillator strengths of f $\sim 0.5$ and $\mathrm{f} \sim 1$, respectively (Malkin, 1992).

In relation to the triphenylene cation, three gas-phase photoelectron (PES) studies (Boschi et al., 1974; Brogli and Heilbronner, 1972; Schmidt, 1977) of triphenylene yield vertical ionic state separations with corresponding mean excitation wavelengths which we calculate to fall near 690 and $570 \mathrm{~nm}$. Unfortunately, apart from the first photoelectron band (Boschi et al., 1974), no laboratory photoelectron spectra have been published that could assist in making spectral assignments, as noted in the critical analysis of available data by Deleuze (2002). Khan (1978, 1992) reported electronic spectra of the triphenylene cation in boric acid glass covering the 200-900 $\mathrm{nm}$ region and compared these data with both photoelectron spectra and theoretical results. An electronic spectrum of the cation in a low-temperature freon matrix is also known (Shida, 1988) which revealed absorption bands at 1715 and $1360 \mathrm{~nm}$ in the nearinfrared, 708, 688, 640 and $583 \mathrm{~nm}$ in the visible and 405, 397, 385 and $363 \mathrm{~nm}$ in the near-UV. Similar results were obtained for the cation in an s-BuCl matrix (Shida and Iwata, 1973). Finally, magnetic circular dichoism spectra are available for both the cation and the anion in boric glass at high temperature (van Paridon et al., 1979).

As is the case for the benzene cation, the triphenylene cation has two almost isoenergetic ${ }^{2} \mathrm{~A}_{2}$ and ${ }^{2} \mathrm{~B}_{1}$ 'ground' electronic states in $C_{2 v}$ symmetry which are separated in energy due to Jahn-Teller interaction. Keszthelyi et al. (2000) find that QCFF/PI and ROHF calculations predict the ${ }^{2} \mathrm{~A}_{2}$ state to be the lower in energy, whereas DFT methods they employed favour ${ }^{2} \mathrm{~B}_{1}$ as the lower state. It was noted that the 'almost negligible' difference in energy between the states indicates that the cation could be fluxional in nature, but this may not be the case in a low-temperature argon or water matrix, or under interstellar conditions. A later study of these two 'ground' states by Kato and Yamabe (2005) found that their HOMO (B) with $A_{2}$ symmetry is stabilised whereas HOMO (A) with $B_{1}$ symmetry is destabilised by vibronic interaction. We adopt this state ordering here and label the electronic states following the notation of Keszthelyi et al. (2000) as $D_{0}$, $D_{1}$ etc. in order of increasing energy. The symmetry of the transitions is as fol-

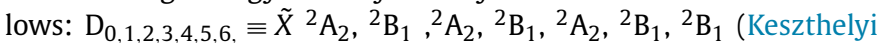
et al., 2000). No gas-phase electronic spectrum of the triphenylene cation is known, but an infrared gas-phase spectrum has been recorded and was found to be complex with significant deviations from theoretical prediction; this is possibly due to Jahn-Teller effects (Oomens et al., 2006). Finally, the QCFF/PI calculations by Keszthelyi et al. (2000) predict absorption wavelengths of 642, 588 and $386 \mathrm{~nm}$ for UV-vis transitions from $\tilde{X}^{2} \mathrm{~A}_{2}$ and 647/641, 595 and $386 \mathrm{~nm}$ from $\tilde{X}^{2} \mathrm{~B}_{1}$, with oscillator strengths in the range 0.03 to 0.1 ; Hirata et al. (2003) obtained bands at 685 and $574 \mathrm{~nm}$ (with $f$ values of 0.06 and 0.116 , respectively) using TD-DFT methods. Although electronic (and vibrational) spectra of a large number of PAH radical cations in low-temperature inert gas matrices have been recorded, to the best of our knowledge there are no reported equivalent studies of the triphenylene cation.

\section{Results}

The neutral triphenylene molecule, $\mathrm{C}_{18} \mathrm{H}_{12}$, and its radical cation have been studied here in relation to their possible presence in water ice mantles on interstellar grains and as potential diffuse interstellar band carriers.

\subsection{Spectra recorded in solid argon and water ice}

A sample of neutral triphenylene was deposited in solid argon as described in Section 3 and yielded the absorption spectrum as shown in Fig. 2(a). Further (sharper) bands near $330 \mathrm{~nm}$ were observed in thicker samples and are attributed to the weak $\left(S_{1} \leftarrow S_{0}\right)$ transition (Levell et al., 2010) (see inset). Exposure to vacuum UV radiation using the MW discharge lamp results in ionisation of triphenylene (ionisation energy of $7.88 \mathrm{eV}$ (Jochims et al., 1999)). The resulting spectrum is shown in Fig. 2(b) and is comparable to that obtained by Shida (1988) at $77 \mathrm{~K}$ in a freon matrix, but suffers significantly less from matrix perturbations (see Table 1).

Embedding neutral triphenylene in water ice results in a matrix shift of roughly $0.5 \mathrm{~nm}$ (at $402 \mathrm{~nm}$ ) to longer wavelengths compared with the argon matrix. This is illustrated in Fig. 3(a), which also shows that the overall triphenylene features in $\mathrm{Ar}$ and $\mathrm{H}_{2} \mathrm{O}$ are rather similar. The resulting spectrum upon VUV irradiation is shown in Fig. 3(b). The signal to noise ratios of the spectra in water 


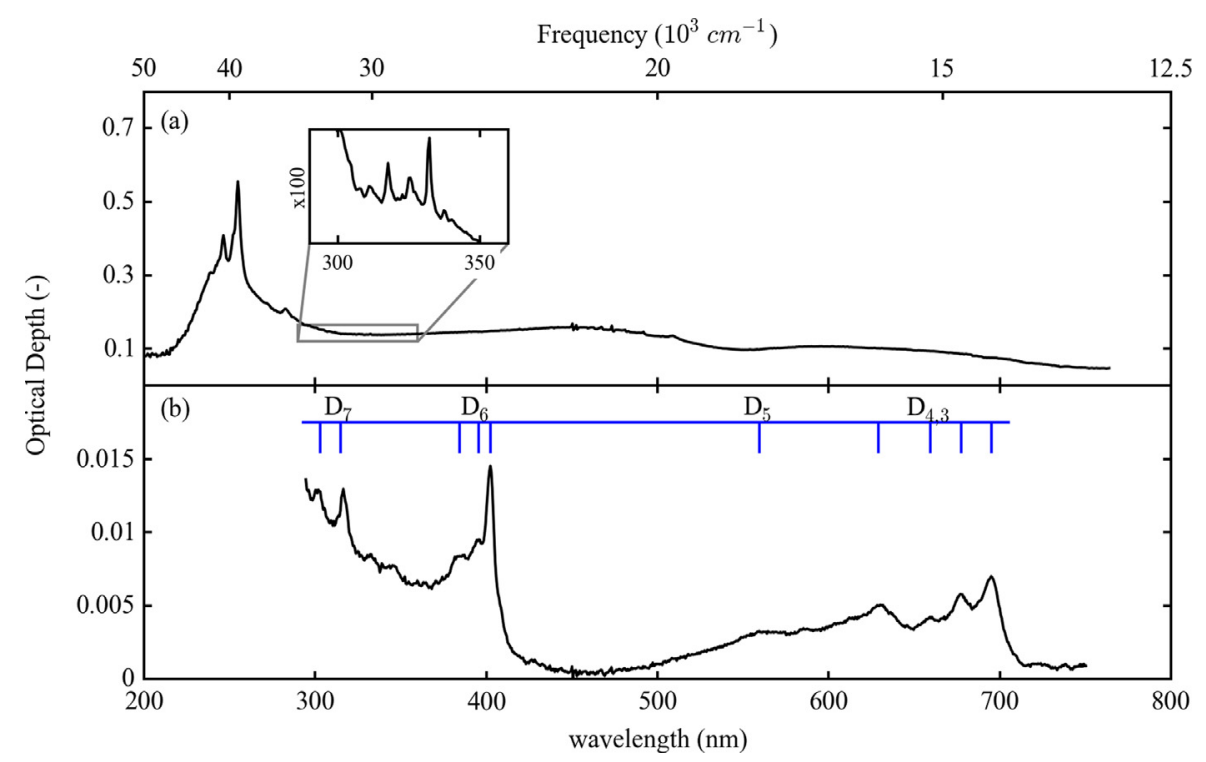

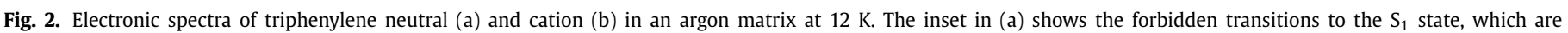

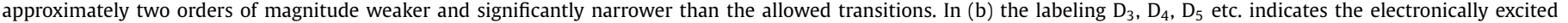
doublet (D) state of the cation involved in the transition and follows the notation given in Keszthelyi et al. (2000).

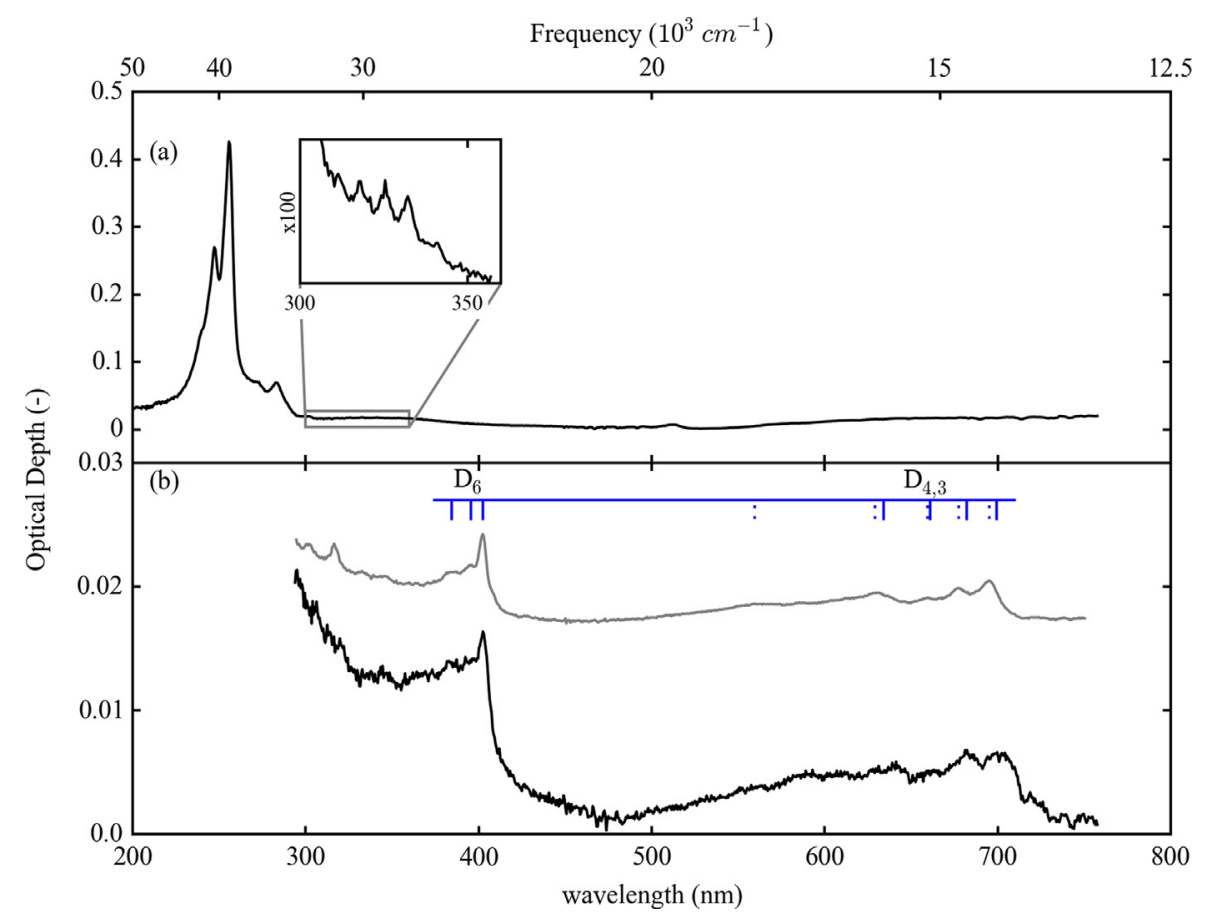

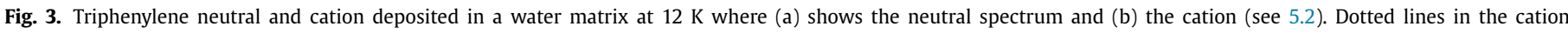

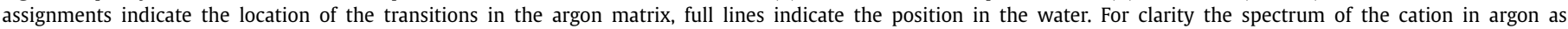
presented in 2 is shown above the cation spectrum in water ice.

are lower as less triphenylene can effectively be ionised due to the limited penetration depth of VUV-radiation in water (see Cruz-Diaz et al., 2014) It should also be noted that the transition strength of the cation is relatively low. Additionally, peak broadening is observed for both the neutral and the cation (see Table 1), which is due to the stronger interactions of water with molecules (as compared to $\mathrm{Ar}$ ).

\subsection{Spectroscopic assignments}

As outlined in Section 4, the electronic symmetry of the ground state of the triphenylene cation has not yet been established with certainty, and could be either ${ }^{2} \mathrm{~B}_{1}$ or ${ }^{2} \mathrm{~A}_{2}$. It is also uncertain as to whether both of these electronic states are populated in a low-temperature matrix. The observed electronic transitions of the cation fall into three main groups near 690 and $560 \mathrm{~nm}$ (broad) and at $402 \mathrm{~nm}$ with some further bands near $300 \mathrm{~nm}$. Guided by the results of calculations and photoelectron spectra (see text and Table 1), these three groups are assigned to transitions from the ground $D_{0}$ state to $D_{3} / D_{4}, D_{5}$ and $D_{6}$ respectively as shown in Fig. 2 and listed in Table 1.

Absorption appears most prominently in two main regions in the argon matrix spectra near 700 and $402 \mathrm{~nm}$. Keszthelyi et al. 
Table 1

Summary of results for neutral triphenylene (I) and the triphenylene cation (II) and literature values for the cation (III).

\begin{tabular}{|c|c|c|c|c|}
\hline $\begin{array}{l}\text { Experimental } \\
\text { I. Triphenylene }\end{array}$ & $\mathrm{T}[\mathrm{K}]$ & Transition & $\lambda[\mathrm{nm}]^{\mathrm{a}}$ & Width $\left[\mathrm{nm}\left(\mathrm{cm}^{-1}\right)\right]^{\mathrm{b}}$ \\
\hline Argon (this work) & 12 & $\begin{array}{l}S_{3} \leftarrow S_{0} \\
S_{2} \leftarrow S_{0} \\
S_{1} \leftarrow S_{0}\end{array}$ & $\begin{array}{l}\frac{255}{282}(246,238) \\
317,325,332\end{array}$ & $2.2(340)$ \\
\hline $\begin{array}{l}\text { Water (this work) } \\
\text { II. Triphenylene }^{+}\end{array}$ & 12 & $\begin{array}{l}S_{3} \leftarrow S_{0} \\
S_{2} \leftarrow S_{0} \\
S_{1} \leftarrow S_{0}\end{array}$ & $\begin{array}{l}\frac{256}{283}(248,240) \\
318,325,332\end{array}$ & $3.4(440)$ \\
\hline Argon (this work) & 12 & $\begin{array}{l}D_{7} \\
D_{6} \\
D_{5} \\
D_{4,3}\end{array}$ & $\begin{array}{l}316(300) \\
\frac{402}{560}(395,384) \\
695,(677,659,629)\end{array}$ & $4(250)$ \\
\hline Water (this work) & 12 & $\begin{array}{l}D_{6} \\
D_{4,3}\end{array}$ & $\left.\frac{402}{699}(682), 661,634\right)$ & $4.5(280)$ \\
\hline Freon $^{c}$ & 77 & not assigned & $\begin{array}{l}\frac{405}{583}(397,385) \\
640 \\
708(688)\end{array}$ & $6(370)$ \\
\hline PES $^{\mathrm{d}}$ & - & $\begin{array}{l}\text { Vertical ionisation } \\
\text { energies (see text) }\end{array}$ & $\begin{array}{l}570 \\
690\end{array}$ & - \\
\hline $\begin{array}{l}\text { Theory } \\
\text { III. Triphenylene }{ }^{+}\end{array}$ & $\mathrm{T}[\mathrm{K}]$ & Transition & $\lambda[\mathrm{nm}]^{\mathrm{e}}$ & Width $\left[\mathrm{nm}\left(\mathrm{cm}^{-1}\right)\right]^{\mathrm{f}}$ \\
\hline $\mathrm{QCFF} / \mathrm{PI}^{\mathrm{g}, \mathrm{h}}$ & - & $\begin{array}{l}{ }^{2} \mathrm{~B}_{1}-\tilde{X}{ }^{2} \mathrm{~A}_{2}\left(D_{6}\right) \\
{ }^{2} \mathrm{~A}_{2}-\tilde{X}{ }^{2} \mathrm{~B}_{1}\left(D_{6}^{\prime}\right) \\
D_{5} \\
D_{5}^{\prime} \\
D_{4}, D_{3} \\
D_{4}^{\prime}, D_{3}^{\prime}\end{array}$ & $\begin{array}{l}386[0.12] \\
386[0.11] \\
588[0.09] \\
595[0.11] \\
642[0.09], 642[0.03] \\
647[0.07], 641[0.04]\end{array}$ & - \\
\hline TD-DFT & - & $\begin{array}{l}\pi_{0}^{*}-\pi_{-5}\left(D_{5}\right) \\
\pi_{0}^{*}-\pi_{-4}\left(D_{4}\right)\end{array}$ & $\begin{array}{l}574[0.116] \\
685[0.060]\end{array}$ & - \\
\hline
\end{tabular}

a The strongest principal absorption bands are underlined and satellite bands are listed in parentheses (). The computed $f$-value where available is given in parentheses [].

b The width is estimated based on the part of the peak that is exposed above the overlapping features. Note that this is not precisely the FWHM, as most of the transitions are not pure Gaussians/Lorentzians.

c Shida (1988).

d Boschi et al. (1974); Brogli and Heilbronner (1972); Schmidt (1977).

e The strongest principal absorption bands are underlined and satellite bands are listed in parentheses (). The computed $f$-value where available is given in parentheses [].

${ }^{f}$ The width is estimated based on the part of the peak that is exposed above the overlapping features. Note that this is not precisely the FWHM, as most of the transitions are not pure Gaussians/Lorentzians.

g Keszthelyi et al. (2000).

h It should be noted that the 'ground' ${ }^{2} \mathrm{~A}_{2}$ and ${ }^{2} \mathrm{~B}_{1}$ states have almost the same energy. Transitions near $5 \mu \mathrm{m}\left(\right.$ to $\left.D_{1}\right)$ are also predicted but with extremely low oscillator strengths of $0.0007 / 0.0001$ and are neglected here, as are transitions to the $\mathrm{D}_{2}$ state predicted at $1280 \mathrm{~nm}$.

i Hirata et al. (2003).

(2000) calculated the energies of the relevant electronic states at optimised geometries for the ${ }^{2} \mathrm{~B}_{1}$ and ${ }^{2} \mathrm{~A}_{2}$ states, and also computed the oscillator strengths for the relevant transitions (see Table 1). The $402 \mathrm{~nm}$ band of the triphenylene cation in argon is most readily assigned to the calculated origin bands which are both predicted to fall at $386 \mathrm{~nm}$ with oscillator strengths of 0.11 and are due to ${ }^{2} \mathrm{~A}_{2}-\tilde{X}^{2} \mathrm{~B}_{1}$ or ${ }^{2} \mathrm{~B}_{1}-\tilde{X}^{2} \mathrm{~A}_{2}$, depending on the ground state adopted. The additional weaker bands located at 395 and $384 \mathrm{~nm}$ probably arise from excitation to vibrationally excited states which lie about 440 and $1160 \mathrm{~cm}^{-1}$ higher in energy, respectively.

Vibrational excitation of $440 \mathrm{~cm}^{-1}$ probably corresponds to promotion of the mode with $\mathrm{a}_{1}$ symmetry $\left(419 \mathrm{~cm}^{-1}\right)$ which was identified with the strongest band in the resonance Raman spectrum of the triphenylene cation by Keszthelyi et al. (2000), and is illustrated in Fig. 4 of their paper. We report an additional absorption feature at $316 \mathrm{~nm}\left(D_{7}\right)$, with a possible vibronic component at $300 \mathrm{~nm}$, which was not reported in the studies of Keszthelyi et al. (2000).

In contrast the 695 - $629 \mathrm{~nm}$ spectral region is more complex and involves fully allowed photo-excitation to two excited electronic states, each potentially with associated vibrational band structure. This might be disentangled through computation of the vibrational level structure in the excited electronic states.

Finally we note that, while not recorded in this work, weaker bands near 1715 and $1360 \mathrm{~nm}$, recorded by Shida (1988) using a freon matrix and attributed to the triphenylene cation, can reasonably be assigned as arising from excitations to the ${ }^{2} \mathrm{~A}_{2}\left(D_{2}\right)$ state which is calculated to lie $0.97 \mathrm{eV}$ above the ground state, with a computed oscillator strength of 0.05 (Keszthelyi et al., 2000). 


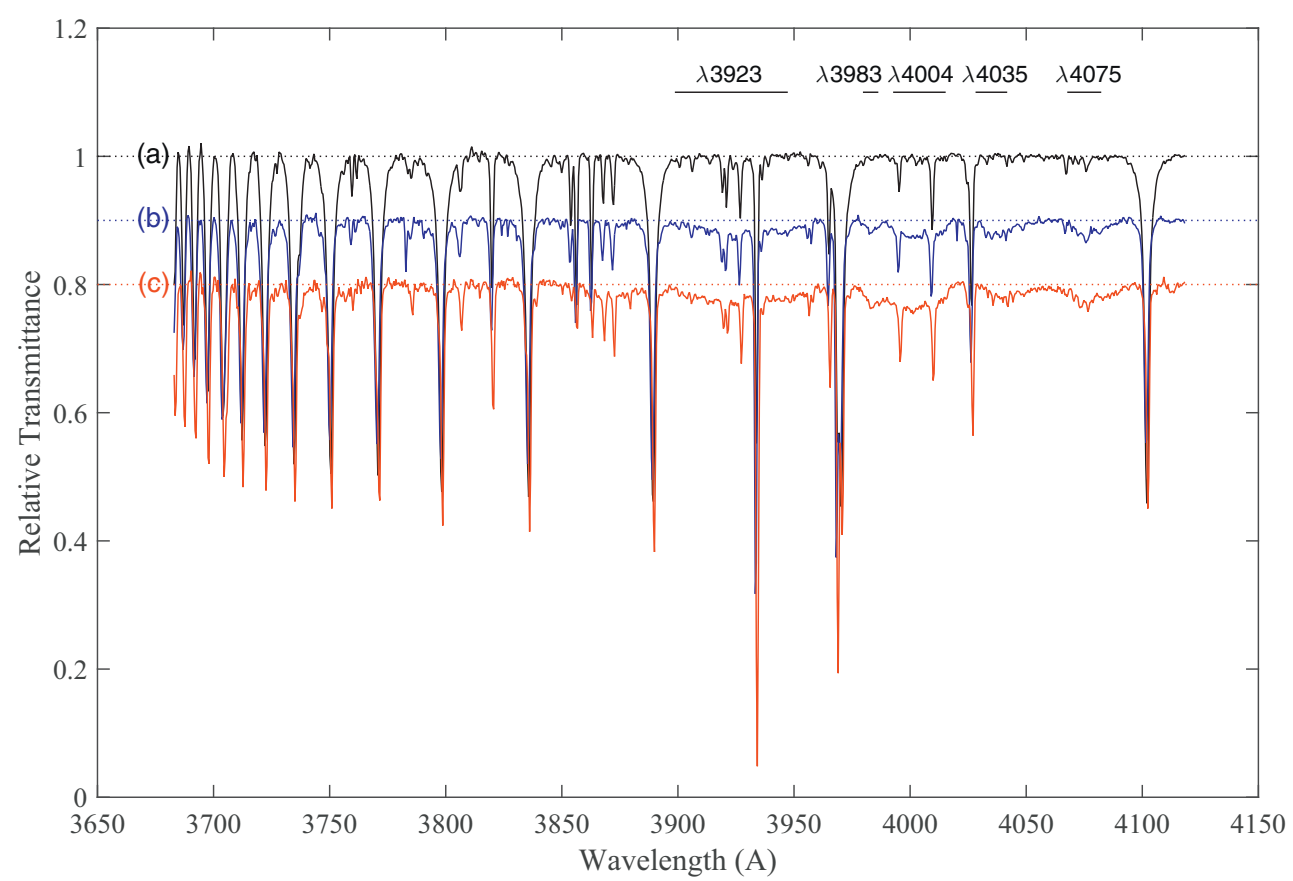

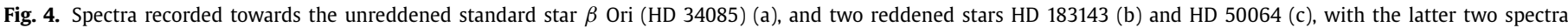

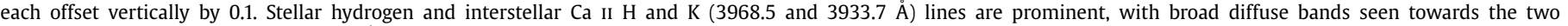
reddened stars between 3850 and $4100 \AA$ (see also Table 3 and the text). The location of the diffuse bands is marked with horizontal bars.

\section{Astrophysical implications}

The motivation behind the water matrix studies is that in relatively dense interstellar regions PAHs are expected to freeze out on dust grains, where they will be embedded in water ice. Given the special nature of the ice, i.e., conserving the charged nature of individual PAHs in an environment where densities can get much higher than in the diffuse interstellar medium, electronic solid state spectra as presented here offer an alternative way to search for individual PAHs in space (Linnartz, 2014), particularly for the case of the triphenylene cation which is considered to be relatively stable. However, observational absorption studies will be challenging, as the number of suitable sight-lines where there is sufficient background light, but not so much as to cause (photo)-evaporation of the ice, is limited. Non-steady-state conditions would help in exposing ice-containing dust to sufficient illumination. An ideal case is an embedded young stellar object with ice-covered grains which is sufficiently dense, but also sufficiently transparent to background light to enable near-UV-vis absorption spectra to be obtained. Spectra as shown in Fig. 3 provide a valuable template for such studies.

PAH radical cations are commonly proposed as carriers of diffuse interstellar bands and a key issue is whether the gas-phase triphenylene cation might be responsible for a (small) part of the diffuse interstellar band spectrum. An estimate of the gas-phase origin bands for this radical cation can be made by comparing well-known laboratory gas-phase and Ar-matrix electronic spectra for other chemically similar PAH radical cations. For the cations of naphthalene $\left(\mathrm{C}_{10} \mathrm{H}_{8}{ }^{+}\right)$(Romanini et al., 1999; Salama and Allamandola, 1991), phenanthrene $\left(\mathrm{C}_{14} \mathrm{H}_{10}{ }^{+}\right)$(Andrews et al., 1985; Pino et al., 1999), acenaphthene $\left(\mathrm{C}_{12} \mathrm{H}_{10}{ }^{+}\right)$(Banisaukas et al., 2003; Biennier et al., 2003), anthracene $\left(\mathrm{C}_{14} \mathrm{H}_{10}{ }^{+}\right)$(Sukhorukov et al., 2004; Szczepanski et al., 1993), coronene $\left(\mathrm{C}_{24} \mathrm{H}_{12}{ }^{+}\right)$(Hardy et al., 2017; Szczepanski and Vala, 1993) and pyrene $\left(\mathrm{C}_{16} \mathrm{H}_{10}{ }^{+}\right)$(Biennier et al., 2004; Salama and Allamandola, 1992), the shifts to lower wavenumber induced by the argon matrix are 95, 98, 223, 266, 232 and $352 \mathrm{~cm}^{-1}$, respectively; the corresponding gas-phase line widths in jet-cold spectra are all quite broad, being $25,16,55$, < 94,106 and $145 \mathrm{~cm}^{-1}$, respectively. Hence for cationic triphenylene, using these laboratory data and the Ar-matrix data in Table 1, the gas-phase origins are predicted to fall in the $3970-4010 \AA$ and 6780 - $6900 \AA$ regions. In practice the latter $6780-6900 \AA$ region is far too congested with diffuse bands and telluric features for a meaningful comparison between laboratory and astronomical data to be made.

However, a more tractable question is whether there exist broad interstellar absorption features in the 3970 - $4010 \AA$ spectral region? This part of the interstellar absorption spectrum has been little studied and presents challenges as discussed by Hobbs et al. (2008, 2009). These include a relatively poor sensitivity level as the photon flux is low due to interstellar reddening, the presence of atomic hydrogen photospheric lines, and a selection effect which discriminates against broad features and reduces their detectability; this is strongest for high-resolution observations, as is the case for most diffuse band surveys, resulting in a low central depth for a broad absorption band even when its equivalent width is reasonably high (Hobbs et al., 2008, 2009).

A summary on the few near-UV studies of interstellar absorption spectra undertaken up to 1995 has been given by Herbig (1995). These include recordings made using a photoelectric scanner at a resolution of $20 \AA$ by Honeycutt (1972) who found absorption between c. 3850 and $4100 \AA$ but could not be certain that it was interstellar in origin. More recent surveys covering the near UV (see Sonnentrucker (2014) and references therein) have generally been conducted at high spectroscopic resolution making detection of broad features difficult. A good discussion of Apache Point Observatory (APO)-based observations, together with those of Jenniskens and Désert (1994) and of Tuairisg et al. (2000) - all of which cover the near-UV, is presented in Hobbs et al. (2008; 2009). It is recognised by Hobbs and coworkers that their studies were biased against detection of broad DIBs, as was noted for their separate study by Jenniskens and Désert (1994). A dedicated programme of low-resolution APO observations designed to address this issue has been initiated (York et al., 2014), and other 
Table 2

Spectral type and $E_{B-V}$ for observed stars.

\begin{tabular}{lll}
\hline Star & Spectral type & $\mathrm{E}_{\mathrm{B}-\mathrm{V}}$ \\
\hline HD 50064 & B6Ia & 0.82 \\
HD 183143 & B7Ia & 1.26 \\
$\beta$ Ori & B8Ia & 0
\end{tabular}

\section{Table 3}

Diffuse band features recorded in the near-UV spectral region towards HD 183143. The measured rest wavelength, full width at half maximum (FWHM), central depth $\left(\mathrm{A}_{c}\right)$ and equivalent width $(\mathrm{W})$ are listed. The two bands marked* are probably interstellar in origin; the one at 3983 A was listed as a possible diffuse band by Hobbs et al. (2009) at $3983.60 \AA$ A with a FWHM of $5.3 \AA$

\begin{tabular}{lllll}
\hline Band & $\lambda_{\text {rest }} / \AA ;$ & FWHM/Å; & $\mathrm{A}_{\mathrm{c}}$ & $\mathrm{W} / \mathrm{m} \AA ;$ \\
\hline$\lambda 4075$ & $4075(2)$ & $14(2)$ & $0.020(2)$ & $280(40)$ \\
$\lambda 4035$ & $4035(2)$ & $13(2)$ & $0.018(2)$ & $270(30)$ \\
$\lambda 4004$ & $4004(2)$ & $22(3)$ & $0.026(2)$ & $540(40)$ \\
$\lambda 3983^{*}$ & $3983(1)$ & $6(1)$ & $0.020(2)$ & $110(25)$ \\
$\lambda 3923^{*}$ & $3923(4)$ & $48(8)$ & $0.021(2)$ & $930(70)$ \\
\hline
\end{tabular}

major surveys covering the near-UV have been recently initiated (Maíz Apellániz, 2015). Observational data for a number of reddened O- and B-type and comparison stars were obtained by Herbig and Sarre in 1992 and 1993 using the 88" University of Hawaii telescope (see Table 2) with the Coudé $\mathrm{f} / 34$ spectrograph, at a far lower resolution of $c$. 7,000. A few diffuse bands in the near-UV were found which are broad with full-width-half-maxima in the range 10-30 $\AA\left(60-200 \mathrm{~cm}^{-1}\right)$ - see Hibbins et al. (1994), Hibbins (1996) and discussion in Herbig (1995). Figure 4 shows for the first time the recorded spectra towards HD 183143 and HD 50064, together with the spectrum of the standard star $\beta$ Ori for comparison. Comparison of the reddened and unreddened spectra reveals the presence of the near-UV bands listed in Table 3 and highlighted in Figure 4. Interestingly these bands all fall in the 3850-4100 $\AA$ range suspected previously by Honeycutt (1972) of being interstellar in origin.

Given that absorption by gas-phase $\mathrm{C}_{18} \mathrm{H}_{12}{ }^{+}$in the $400 \mathrm{~nm}$ region is predicted, this molecule can be considered a candidate for one or more of the broad diffuse bands seen, though gas-phase spectra of the ion are essential to confirm or refute this suggestion. The equivalent widths and FWHM of the measured features towards HD 183143 are given in Table 3.

Taking $300 \mathrm{m \AA}$ as a typical equivalent width (see Table 3), a wavelength of $400 \mathrm{~nm}$, and the computed oscillator strength for the ${ }^{2} \mathrm{~A}_{2}-\tilde{X}$ transition of 0.11 (Keszthelyi et al., 2000), it is possible to estimate the column density of the triphenylene cation towards HD 183143 that would be required to produce such an absorption. The column density obtained is $2 \times 10^{13} \mathrm{~cm}^{-2}$ which is similar to the value inferred for $\mathrm{C}_{60}{ }^{+}$(Campbell et al., 2016b), though the triphenylene cation would make a demand on the carbon budget which is lower by a factor of three. It is also expected that as the bands would be from a PAH cation, the spectral linewidths would be larger than for transitions of neutral PAHs and this is corroborated by the gas-phase experimental data for similar PAH cations. For reference, a FWHM of $145 \mathrm{~cm}^{-1}$ (as measured in the laboratory for the similar-sized gas-phase pyrene cation) corresponds to $23 \AA$ at $400 \mathrm{~nm}$ which is similar to the widths of the absorption features under discussion (see Table 3). We conclude that there is observational evidence of interstellar absorption bands in the $400 \mathrm{~nm}$ region that are consistent in wavelength and width with those expected for the gas-phase triphenylene cation, but that gas-phase laboratory spectra are needed, particularly as the absorption bands are expected to be broad.

\section{Acknowledgements}

Financial support through the NWO program PEPSci (Planetary and ExoPlanetary Science) and a NWO VICI grant is acknowledged. PJS thanks the Leverhulme Trust for award of a Research Fellowship and Leiden Observatory for hospitality during the course of this work. We wish to record our appreciation of the interest and encouragement of the late George Herbig and of his contribution to the astronomical observations described here.

\section{References}

Allamandola, L.J., Tielens, A.G.G.M., Barker, J.R., 1989. Astrophys. J. Suppl. Ser. 71, 733-775.

Allodi, M.A., Baragiola, R.A., Baratta, G.A., Barucci, M.A., Blake, G.A., Boduch, P. Brucato, J.R., Contreras, C., Cuylle, S.H., Fulvio, D., Gudipati, M.S., Ioppolo, S., Kaňuchová, Z., Lignell, A., Linnartz, H., Palumbo, M.E., Raut, U., Rothard, H., Salama, F., Savchenko, E.V., Sciamma-O’Brien, E., Strazzulla, G., 2013. Space Sci. Rev. 180, 101-175.

Andrews, L., Friedman, R.S., Kelsall, B.J., 1985. J. Phys. Chem. 89, 4016-4020.

Banisaukas, J., Szczepanski, J., Eyler, J., Vala, M., Hirata, S., Head-Gordon, M., Oomens, J., Meijer, G., von Helden, G., 2003. J. Phys. Chem. A 107, 782-793.

Baratta, G.A., Palumbo, M.E., 1998. J. Opt. Soc. Am. A 15, 3076-3085.

Berné, O., Montillaud, J., Joblin, C. , 2015. Astron. Astrophys. 577, A133.

Berné, O., Montillaud, J., Joblin, C., 2016. Astron. Astrophys, 588, C1.

Berné, O., Mulas, G. Joblin, C., 2013. Astron. Astrophys, 550, L4.

Bernstein, M.P., Sandford, S.A., Allamandola, L.J., 2005. Astrophys. J. Suppl. Ser. 161, 53-64.

Bernstein, M.P., Sandford, S.A., Mattioda, A.L., Allamandola, L.J., 2007. Astrophys. J. $664,1264-1272$

Bhatt, N.H., Cami, J. , 2015. Astrophys. J. Suppl. Ser. 216, 22.

Biennier, L., Salama, F., Allamandola, L.J., Scherer, J.J., 2003. J. Chem. Phys. 118 7863-7872.

Biennier, L., Salama, F., Gupta, M., O’Keefe, A., 2004. Chem. Phys. Lett. 387, 287-294. Boschi, R., Clar, E., Schmidt, W., 1974. J. Chem. Phys. 60, 4406-4418.

Bossa, J.-B., Isokoski, K., Paardekooper, D.M., Bonnin, M., van der Linden, E.P., Triemstra, T., Cazaux, S., Tielens, A.G.G.M., Linnartz, H., 2014. Astron. Astrophys. 561, A136.

Bouwman, J., Mattioda, A.L., Linnartz, H., Allamandola, L.J., 2011. Astron. Astrophys. 525, A93.

Bouwman, J., Paardekooper, D.M., Cuppen, H.M., Linnartz, H., Allamandola, L.J., 2009. Astrophys. J. 700, 56-62.

Brogli, F., Heilbronner, E., 1972. Angew. Chem. Int. Ed. 11, 538.

Callahan, M.P., Abo-Riziq, A., Crews, B., Grace, L., de Vries, M.S., 2008. Spectrochim. Acta Mol. Biomol. Spectrosc. 71, 1492-1495

Cami, J., Bernard-Salas, J., Peeters, E., Malek, S.E., 2010. Science 329, 1180.

Campbell, E.K., Holz, M., Gerlich, D., Maier, J.P., 2015. Nature 523, 322-323.

Campbell, E.K., Holz, M., Maier, J.P., 2016a. Astrophys. J. Lett. 826, L4.

Campbell, E.K., Holz, M., Maier, J.P., Gerlich, D., Walker, G.A.H., Bohlender, D., 2016b. Astrophys. J. 822, 17.

Candian, A., Sarre, P.J., Tielens, A.G.G.M., 2010. Astrophys. J. Lett. 791, L10.

Cook, A.M., Ricca, A., Mattioda, A.L., Bouwman, J., Roser, J., Linnartz, H., Bregman, J. Allamandola, L.J., 2015. Astrophys. J. 799, 14

Cordiner, M.A., Cox, N.L.J., Lallement, R., Najarro, F., Cami, J., Gull, T.R., Foing, B.H., Linnartz, H., Lindler, D.J., Proffitt, C.R., Sarre, P.J., Charnley, S.B., 2017. ArXiv e-prints.

Cruz-Diaz, G.A., M., M.G., Chen, Y.-J., Yih, T.-S., 2014. Astron. Astrophys. 562, A119.

Cuylle, S.H., Allamandola, L.J., Linnartz, H., 2014. Astron. Astrophys. 562, A22.

Deleuze, M.S., 2002. J. Chem. Phys. 116, 7012-7026.

Foing, B.H., Ehrenfreund, P., 1994. Nature 369, 296-298.

Fulara, J., Jakobi, M., Maier, J.P., 1993. Chem. Phys. Lett. 211, 227-234.

Galazutdinov, G.A., Shimansky, V.V., Bondar, A., Valyavin, G., Krełowski, J., 2017. Mon. Not. R. Astron. Soc 465, 3956-3964.

Gredel, R., Carpentier, Y., Rouillé, G., Steglich, M., Huisken, F., Henning, T., 2011. Astron. Astrophys. 530, A26.

Gudipati, M.S., Allamandola, L.J., 2003. Astrophys. J. Lett. 596, L195-L198.

Gudipati, M.S., Allamandola, L.J., 2004. Astrophys. J. Lett. 615, L177-L180.

Gudipati, M.S., Allamandola, L.J. 2006. Astrophys. J. 638, 286-292.

Guennoun, Z., Aupetit, C., Mascetti, J., 2011. Phys. Chem. Chem. Phys. 13, 7340-7347.

Hardy, F.-X., Rice, C.A., Maier, J.P., 2017. Astrophys. J. 836, 37.

Herbig, G.H., 1995. Annu. Rev. Astron. Astrophys. 33, 19-74.

Hibbins, R.E., 1996. The University of Nottingham.

Hibbins, R.E., Miles, J.R., Sarre, P.J., Herbig, G.H., 1994. In: Tielens, A.G.G.M. (Ed.), The Diffuse Interstellar Bands, p. 31.

Hirata, S., Head-Gordon, M., Szczepanski, J., Vala, M., 2003. J. Phys. Chem. A 107, 4940-4951.

Hobbs, L.M., York, D.G., Snow, T.P., Oka, T., Thorburn, J.A., Bishof, M., Friedman, S.D. McCall, B.J., Rachford, B., Sonnentrucker, P., Welty, D.E., 2008. Astrophys. J. 680, 1256-1270.

Hobbs, L.M., York, D.G., Thorburn, J.A., Snow, T.P., Bishof, M., Friedman, S.D., McCall, B.J., Oka, T., Rachford, B., Sonnentrucker, P., Welty, D.E., 2009. Astrophys. J. 705, 32-45. 
Honeycutt, R.K., 1972. Astron. J. 77, 24-28.

Hudgins, D.M., Sandford, S.A., Allamandola, L.J., Tielens, A.G.G.M., 1993. Astrophys. J. Suppl. Ser. 86, 713-870.

Huisken, F., Rouillé, G., Steglich, M., Carpentier, Y., Jäger, C., Henning, T., 2014. The Diffuse Interstellar Bands. In: Cami, J., Cox, N.L.J. (Eds.). In: IAU Symposium, 297, pp. 265-275.

Jenniskens, P., Désert, F.-X., 1994. Astron. Astrophys. Suppl. Ser. 106, 39-78.

Jochims, H.W., Baumgärtel, H., Leach, S., 1999. Astrophys. J. 512, 500-510.

Kato, T., Yamabe, T., 2005. Chem. Phys. Lett. 403, 113-118.

Keszthelyi, T., Balakrishnan, G., Wilbrandt, R., Yee, W.A., Negri, F., 2000. J. Phys. Chem. A 104, 9121-9129.

Khan, Z., 1978. Can. J. Spectrosc. 23, 8-15.

Khan, Z., 1992. Acta. Phys. Pol. A 82, 937-955.

Kjaergaard, H.G., Robinson, T.W., Brooking, K.A., 2000. J. Phys. Chem. A 104, 11297-11303.

Kokkin, D.L., Reilly, N.J., Troy, T.P., Nauta, K., Schmidt, T.W., 2007. J. Chem. Phys. 126. 084304-084304

Kuhn, M., Renzler, M., Postler, J., Ralser, S., Spieler, S., Simpson, M., Linnartz, H., Tielens, A.G.G.M., Cami, J., Mauracher, A., Wang, Y., Alcamí, M., Martín, F., Beyer, M.K., Wester, R., Lindinger, A., Scheier, P., 2016. Nat. Commun. 7, 13550.

Levell, J.W., Ruseckas, A., Henry, J.B., Wang, Y., Stretton, A.D., Mount, A.R., Galow, T.H., Samuel, I.D.W., 2010. J. Phys. Chem. A 114, 13291-13295.

Ligterink, N.F.W., Paardekooper, D.M., Chuang, K.-J., Both, M.L., Cruz-Diaz, G.A., van Helden, J.H., Linnartz, H., 2015. Astron. Astrophys. 584, A56.

Linnartz, H., 2014. The Diffuse Interstellar Bands. In: Cami, J., Cox, N.L.J. (Eds.). In: IAU Symposium, 297, pp. 359-363.

Maíz Apellániz, J., 2015. Mem. Soc. Astron. Ital. 86, 553.

Malkin, J., 1992. CRC press.

Oomens, J., Sartakov, B.G., Meijer, G., von Helden, G., 2006. Int. J. Mass Spectrom. 254, 1-19.

Pino, T., Boudin, N., Bréchignac, P., 1999. J. Chem. Phys, 111, 7337-7347.

Romanini, D., Biennier, L., Salama, F., Kachanov, A., Allamandola, L.J., Stoeckel, F., 1999. Chem. Phys. Lett. 303, 165-170.

Salama, F., 2008. Organic Matter in Space. In: Kwok, S., Sanford, S. (Eds.). In: IAU Symposium, 251, pp. 357-366.

Salama, F., Allamandola, L.J., 1991. J. Chem. Phys. 94, 6964-6977.

Salama, F., Allamandola, L.J., 1992. Nature 358, 42.
Salama, F., Ehrenfreund, P., 2014. The Diffuse Interstellar Bands. In: Cami, J., Cox, N.L.J. (Eds.). In: IAU Symposium, 297, pp. 364-369.

Salama, F., Galazutdinov, G., Krełowski, J., Biennier, L., Beletsky, Y., Song, I.O., 2011 The Molecular Universe. In: Cernicharo, J., Bachiller, R. (Eds.). IAU Symposium, 280.

Sandford, S.A., Bernstein, M.P., Allamandola, L.J., 2004. Astrophys. J. 607, 346-360.

Schmidt, W., 1977. J. Chem. Phys. 66, 828-845.

Sellgren, K., Werner, M.W., Ingalls, J.G., Smith, J.D.T., Carleton, T.M., Joblin, C., 2010. Astrophys. J. Lett. 722, L54-L57.

Shida, T., 1988. Elsevier.

Shida, T., Iwata, S., 1973. J. Am. Chem. Soc. 95, 3473.

Sonnentrucker, P., 2014. The Diffuse Interstellar Bands. In: Cami, J., Cox, N.L.J. (Eds.) In: IAU Symposium, 297, pp. 13-22.

Steglich, M., Bouwman, J., Huisken, F., Henning, T., 2011. Astrophys. J. 742, 2.

Sukhorukov, O., Staicu, A., Diegel, E., Rouillé, G., Henning, T., Huisken, F., 2004. Chem. Phys. Lett. 386, 259-264.

Szczepanski, J., Vala, M., 1993. Astrophys. J. 414, 646-655.

Szczepanski, J., Vala, M., Talbi, D., Parisel, O., Ellinger, Y., 1993. J. Chem. Phys. 98, 4494-4511.

Tielens, A.G.G.M., 2013. Rev. Mod. Phys. 85, 1021-1081.

Tuairisg, S.ó., Cami, J., Foing, B.H., Sonnentrucker, P., Ehrenfreund, P., 2000. Astron. Astrophys. Suppl. Ser. 142, 225-238.

van Paridon, M.W., Koning, R.E., Zandstra, P.J., 1979. Chem. Phys. Lett. 61, 258-261.

Walker, G.A.H., Bohlender, D.A., Maier, J.P., Campbell, E.K., 2015. Astrophys. J. Lett 812, L8.

Walker, G.A.H., Campbell, E.K., Maier, J.P., Bohlender, D., Malo, L., 2016. Astrophys. J. 831, 130.

Warneck, P., 1962. Appl. Opt. 1, 721-726.

York, B., Sonnentrucker, P., Hobbs, L.M., York, D.G., Friedman, S.D., Dahlstrom, J., Welty, D.E., Snow, T.P., Rachford, B.L., 2014. The Diffuse Interstellar Bands. In Cami, J., Cox, N.L.J. (Eds.). In: IAU Symposium, 297, pp. 138-140.

Zhen, J., Castellanos, P., Paardekooper, D.M., Ligterink, N., Linnartz, H., Nahon, L. Joblin, C., Tielens, A.G.G.M., 2015. Astrophys. J. Lett. 804, L7.

Zhen, J., Paardekooper, D.M., Candian, A., Linnartz, H., Tielens, A.G.G.M., 2014a. Chem. Phys. Lett. 592, 211-216.

Zhen, J., Castellanos, P., Paardekooper, D.M., Linnartz, H., Tielens, A.G.G.M., 2014b. Astrophys. J. Lett. 797, L30. 